\title{
Network Reconfiguration for Minimizing Power Loss by Moth Swarm Algorithm
}

\author{
Thuan Thanh Nguyen ${ }^{1}$, Duong Thanh Long ${ }^{2 *}$ \\ Faculty of Electrical Engineering Technology \\ Industrial University of Ho Chi Minh City \\ Ho Chi Minh, Viet Nam
}

\begin{abstract}
This paper presents a network reconfiguration approach for minimizing power loss of the distribution system based on moth swarm algorithm (MSA). The MSA is a recent metaheuristic inspired from the navigational technique of moths in the dark for finding food sources. For searching optimal solution, MSA used three different mechanisms of generating new solutions consisting of Lévy-flights, Gaussian walks and spiral flight. The effectiveness of MSA is validated on two distribution systems consisting of the 33-nodes and 69-nodes. The simulation results are compared to particle swarm optimization and other available approaches in the literature. The calculated results on the test systems show that MSA can be an effective and reliable tool for the NR problems.
\end{abstract}

Keywords-Network reconfiguration; moth swarm algorithm; power loss; particle swarm optimization; distribution system

\section{INTRODUCTION}

Network reconfiguration (NR) is a method of finding the optimal radial topology of the distribution system (DS) to reach the goals related to economic or technical benefits. It is performed by changing the status of switches located in the DS. Due to huge benefits of NR technique, it has attracted lot of concerns of researchers for finding the efficient solving method.

The first NR solving method is based on the branch-andbound technique [1]. In which, to find the optimal network configuration for power loss reduction, each switch on closed network is opened to form the radial network reconfiguration. Then many different methods have been proposed for the NR problem, especially techniques based on metaheuristic algorithms. In [2],[3], genetic algorithm (GA) has been proposed to find the optimal network configuration for minimizing power loss of the DS. In which the branches and switches are encoded in the solution vector in [2] while in [3] only open switches are shown in the genetic string of GA. In [4], particle swarm optimization (PSO) is successful applied for the NR problem to find the optimal radial topology for power loss reduction. Similar to [4], PSO is also used as searching tool for finding network configuration in [5], [6]. The NR problem for loss reduction is also solved by artificial bee colony algorithm (ABC) [7]. In this study, the effectiveness of $\mathrm{ABC}$ has been demonstrated by the compared results with other approaches available in the literature. In [8], ant colony search (ACO) is used to determine the optimal network configuration to reduce losses and enhance load balancing of the DS. In addition to the aforementioned popular algorithms which have been successfully applied to the NR problem, some reconfiguration methods based on newly developed algorithms or improved versions of the well-known methods have also been proposed and achieved good results such as improved cuckoo search algorithm (ICSA) [9], fireworks algorithm (FWA) [10], biogeography based optimization (BBO) [11], modified particle swarm optimization (MPSO) [12], improved selective binary PSO (ISBPSO) [13], modified flower pollination algorithm (MFPA) [14], invasive weed optimization (IWO) [15], adaptive shuffled frogs leaping algorithm (ASFLA) [16], binary particle swarm gravity search algorithm (BPSOGSA) [17], harmony search algorithm (HSA) [18], [19], improved adaptive imperialist competitive algorithm (IAICA) [20], and grey wolf optimization (GWO) [21].

It can be seen that the NR problem can satisfy various technical goals such as loss reduction, load balancing, feeder balancing, voltage improvement and reliability enhancing, etc. In particular, loss reduction is one of the most important goal in operating the distribution network because the power loss of the distribution network is often higher than the losses of other parts of the power system. In terms of the method of solving the NR problem, finding new approaches to enrich the search tools of optimal network configuration is still a worthy of concern as the development of the field of optimization is more powerful and more and more good algorithms have been born.

Moth swarm algorithm (MSA) is a recent metaheuristic inspired from the navigational technique of moths in the dark for finding food sources [22], [23]. The moths fly in the right direction in the dark based on light sources like stars or moons. To apply MSA for the optimization problem, position of a light source and its luminescence intensity are considered as a candidate solution and the quality of the candidate solution. Furthermore, the population of moths is divided into three groups consisting of pathfinders, prospectors and onlookers with different roles for exploring and exploiting the search space. In [22], MSA has shown advantages compared with PSO as well as other methods for the optimal power flow problem but the application of MSA for other problems related to power system like the NR problem is still a question.

In this study, MSA is adapted to find the optimal network configuration with minimizing power loss. The NR method based on MSA is evaluated on the 33-nodes and 69-nodes distribution networks. The calculated results are compared with PSO and other available approaches in the literature. The main contributions of the paper can be summarized as follows:

*Corresponding Author 
- MSA is successful adapted to solve the NR problem for minimizing power loss.

- MSA is better than PSO for finding the optimal network configuration with higher successful rate and better quality of obtained solution.

- MSA is able to determine the better network configuration compared to some available approaches in the literature.

The structure of the paper is organized as follows: The mathematical formulation of the NR problem is presented in the Section 2 meanwhile the MSA for solving the NR problem is shown in Section 3. Section 4 presents the performance of MSA on the test systems. Finally, the conclusion is demonstrated.

\section{MATHEMATICAL FORMULATION OF NETWORK RECONFIGURATION}

The power loss (Ploss) of the distribution system (DS) is determined by sum of loss of lines in the DS as follows:

Ploss $=\sum_{i=1}^{n_{l i}} \Delta P_{i}$

In which, $\Delta P_{i}$ is power loss of the line $i . n_{l i}$ is number of lines of the DS.

The network configuration obtained has to satisfy the below constraints:

Constraint of radial topology: This condition is maintained by the following formula [24]:

$|\operatorname{det}(M)|=1$

In which, $\operatorname{det}(M)$ is the $\mathrm{M}$ matrix's determinant. $M$ is a line by node matrix that is built by relationship among lines and nodes of the DS.

Constraints of voltage and current:

$\left\{V_{l o} \leq V_{j} ; j=1, \ldots, n_{n o}\right.$

$\left\{I_{i} \leq I_{i, h i} ; i=1, \ldots, n_{l i}\right.$

In which, $V_{j}$ is the voltage magnitude of the node $j . V_{l o}$ is the allowed minimum voltage magnitude that is usually chosen to 0.95 p.u. $n_{n o}$ is the number of nodes of the DS. $I_{i}$ and $I_{i, h i}$ are the current and rated current of the line $i$.

\section{MOTH SWARM ALGORITHM FOR NETWORK RECONFIGURATION}

The moths transit through a space relying on the angle between a light source and their flying direction. Therefore, in MSA, position of each moth is also considered as a candidate solution. Furthermore, the population of moths is divided into three groups consisting of pathfinders, prospectors and onlookers. In which, the first group has a role of exploring the search space to determine light sources for guiding the warm. The second one has a role of hiking the area that is the neighborhood of the light sources. The final group tends to move slowly to the light source obtained by the second group.

The application of MSA for the NR problem is described in more details as following:
Step 1: Generate randomly new solutions

In order to apply MSA for the NR problem, each network configuration is considered as the position of moths in the search space. Thus, the population of solutions are produced randomly as follows:

$M o_{i, j}=\operatorname{round}\left(M o_{l o, j}+r d_{1} \cdot\left(M o_{h i, j}-M o_{l o, j}\right)\right)$

Where $\mathrm{Mo}_{i, j}$ is the value of the variable $j$ with $j=1,2, \ldots$, $d$ of the solution $i$ with $i=1,2, \ldots, n$. The $d$ and $n$ are dimension of the problem and size of population. $r d_{1}$ is result of the function of rand $[0,1] . \mathrm{Mo}_{h i, j}$ and $\mathrm{Mo}_{l o, j}$ are the upper and lower boundaries of the variable $j$.

The quality of each moth in the population is calculated by using the fitness function as follows:

$\mathrm{f}_{i}=$ Ploss $+K_{p} \cdot\left[\left(\max \left(V_{\min }-V_{l o}\right), 0\right)+\left(\max \left(\max \left(I_{i}\right)\right.\right.\right.$

$\left.\left.\left.\left.I_{i, h i}\right)-1\right), 0\right)\right]$

Where $K_{p}$ is penalty coefficient. $V_{\min }$ is the minimum voltage amplitude of the DS.

Based on the moths' quality, $n_{p a}$ best moths are selected to become pathfinders or called light sources and the rest moths will be considered as prospectors and onlookers follow descending order of quality.

Step 2. Generate new solutions for updating pathfinders

All of pathfinders are updated in this step but not of all variables of each pathfinder is renewed. The variables chosen for updating by the following technique. Firstly, the variation coefficient of all variables in the dimension $j$ of the pathfinders $\left(\mathrm{cv}_{j}\right)$ is determined as follows:

$\mathrm{cv}_{j}=\frac{\sqrt{\frac{1}{n_{p a}} \sum_{i=1}^{n_{p a}}\left(M o_{i, j}-\frac{1}{n_{p a}} \sum_{i=1}^{n_{p a}} M o_{i, j}\right)^{2}}}{\frac{1}{n_{p a}} \sum_{i=1}^{n_{p a}} M o_{i, j}}$

The average value of variation coefficients is calculated as below:

$\mathrm{cv}_{\text {mean }}=\frac{1}{d} \sum_{j=1}^{d} \mathrm{cv}_{j}$

Then any variable of pathfinder that has the lower variation coefficient compared to the average variation coefficient is selected for renewed. The group of variables $\left(g_{u p}\right)$ for renewing is determined as follows:

$j \in g_{\text {up }} ;$ if $\mathrm{cv}_{j}<\mathrm{cv}_{\text {mean }}$

In order to update the variable that belongs to the group $g_{\text {up }}$, the crossover technique among pathfinders are used as follows:

$M o_{p, \text { new }}=\operatorname{round}\left(M o_{r 1}+L V_{p 1} \cdot\left(M o_{r 2}-M o_{r 3}\right)+\right.$

$\left.L V_{p 2} \cdot\left(M o_{r 4}-M o_{r 5}\right)\right)$

Where $M o_{p, \text { new }}$ are new sub-solution vector of the pathfinder $p$ with $p=1, \ldots, n_{p a} . M o_{r 1}$ to $M o_{r 5}$ are five pathfinders selected randomly. $L V_{p 1}$ and $L V_{p 2}$ are scale factors which are produced by the Lévy-flights as below: 
$L V_{i}=\operatorname{sc} \otimes \operatorname{levy}(\alpha)$

Where $s c$ is a scaling factor that is chosen to $0.01 . \alpha$ is distribution factor in $[0,2] . \otimes$ is the entry-wise multiplications.

Then, the new sub-solution vector of the pathfinder is merged with the rest part of the pathfinder to create new pathfinder as follows:

$M o_{p, n e w, j}=\left\{\begin{array}{l}M o_{p, n e w, j} ; \text { if } j \in g_{u p} \\ M o_{p, j} ; \text { otherwise }\end{array}\right.$

The news pathfinders are evaluated quality by the fitness function. Then, the selection technique is used to retain the better pathfinder as follows:

$M o_{p, \text { new }}= \begin{cases}M o_{p, \text { new }} & ; \text { if } f\left(M o_{p, \text { new }}\right)<f\left(M o_{p}\right) \\ M o_{p} & ; \text { otherwise }\end{cases}$

Step 3. Generate new prospectors

The number of prospectors $\left(n_{p r}\right)$ in the population is not fixed, it depends on the number of iterations performed by the algorithm and is determined as follows:

$n_{p r}=\operatorname{round}\left(\left(n-n_{p a}\right) \cdot\left(1-\frac{g}{G}\right)\right)$

Where $g$ and $G$ are the current iteration and the maximum number of iterations for performing MSA.

Then each prospector in the population is renewed based on the spiral flight technique as follows:

$M o_{i, n e w}=\operatorname{round}\left(\left|M o_{i}-M o_{p}\right| \cdot e^{\varphi} \cdot \cos 2 \pi \varphi+M o_{p}\right)$

Where, $M o_{i, n e w}$ and $M o_{i}$ is the new and current solution $i$ with $i \in\left\{n_{p a}+1, \ldots, n_{p a}+n_{p r}\right\} . \varphi$ is a random number in $\left[\left(-1-\frac{g}{G}\right), 1\right] \cdot M o_{p}$ is a pathfinder selected from the $n_{p a}$ of pathfinders. The probability of the pathfinder $p$ chosen is determined as follows:

$\operatorname{Prob}_{p}=\frac{f_{p}}{\sum_{p=1}^{n_{p} a} f_{p}}$

Based on the new prospectors generated, their quality is evaluated by the fitness function. However, in this step, the selection technique does not used to update the current prospectors because the new prospectors may be chosen to become light sources if they have better quality than the pathfinders. Thus, updating of the current population of moths will be performed at the end of the MSA.

\section{Step 4. Generate new onlookers}

In order to produce new onlookers, the number of onlookers that is determined by (16) is divided into small groups and each group is generated by different techniques. The details of producing new solutions of onlookers are described as follows.

$n_{\text {on }}=n-n_{p a}-n_{p r}$

A half of new onlookers are generated by using Gaussian walks technique as follows:

$M o_{i, \text { new }}=\operatorname{round}\left(M o_{i}+\gamma+\left(r d_{2} \cdot M o_{\text {best }}-r d_{3} \cdot M o_{i}\right)\right)(17$
Where $M o_{i, \text { new }}$ and $M o_{i}$ is the new and current onlooker $i$ with $i=1, \ldots, n_{\text {on }} / 2$. Mo ose is the best so far moth in the population. $r d_{2}$ and $r d_{3}$ are random number in [0,1]. $\gamma$ is a normal stochastic distribution vector that is determined from the Gaussian walks technique as follows:

$\gamma=r d_{4} \otimes N\left(M o_{\text {best }}, \frac{\log g}{g} .\left(M o_{i}-M o_{\text {best }}\right)\right)$

Where $r d_{4}$ is random number vector with length of $d$.

A rest half of new onlookers are generated by the associative learning technique as follows:

$$
\begin{aligned}
& M o_{i, n e w}=\operatorname{round}\left(M o_{i}+\tau \cdot \operatorname{rand}\left(\left[M o_{i}-M o_{i, l o}, M o_{i, h i}-\right.\right.\right. \\
& \left.\left.M o_{i}\right]\right)+2 g / G \cdot r d_{5} \cdot\left(M o_{b e s t}-M o_{i}\right)+(1-g / \\
& \left.G) \cdot r d_{6} \cdot\left(M o_{p}-M o_{i}\right)\right)
\end{aligned}
$$

Where $M o_{i, \text { new }}$ and $M o_{i}$ is the new and current onlooker $i$ with $i=1, \ldots, n_{\text {on }} / 2 . \tau$ is scale factor chosen to $0.001 . M o_{p}$ is a pathfinder selected from the $n_{p a}$ of pathfinders with probability of $\operatorname{Prob}_{p} . M o_{i, l o}$ and $M o_{i, h i}$ are lower and upper boundaries of the moth. $r d_{5}$ and $r d_{6}$ are random number in $[0,1]$.

Based on the new onlookers generated, their quality is evaluated by the fitness function.

Step 5: Update new population and determine the best so far solution.

Based on the quality of the current population and new moths. The moths are sorted in ascending order of the fitness values. The $\mathrm{n}$ top moths are selected to become the new population and the types of moths are determined again. In addition, the best pathfinder of the population is chosen to the best so far solution.

The steps 2 to 5 of MSA are executed continuously until the number of iterations reach to the maximum value $G$. Then the best so far solution is considered as the result of the NR problem.

\section{RESULTS AND DISCUSSION}

In order to find optimal network configuration, the MSA for the NR problem is run in a personal computer with $2.4 \mathrm{Gh}$ of CPU and $4 \mathrm{G}$ of RAM on the Matlab platform. Two DS comprising of the 33-nodes and 69-nodes systems shown in Fig. 1 [25], [26] are used to find the best network configuration. For both of the DS, the penalty coefficient $K_{p}$ in the fitness function equation is set to 1000 . The population of moths are set to 20. The maximum number of iterations is chosen to 150. The MSA is executed in 50 trials, the best network configuration in all of runs are considered as the results of the problem. In this study, the results obtained by the proposed MSA are not only compared to those of previous methods in the literature, but also to the PSO that is coded and performed in the same computer for demonstrating the effectiveness of the MSA. In which, PSO is one of well-known algorithm and it is successful applied to many network reconfiguration problem [4],[5],[6]. 


\section{A. The Optimal Network Reconfigurations}

For the 33-nodes network, the initial network configuration with open switches $\{33-34-35-36-37\}$ that causes power loss (Ploss), minimum voltage amplitude $\left(V_{\text {min }}\right)$ and maximum of current per rate current $\left(I / I_{\text {rate }}\right)_{\max }$ of $202.6863 \mathrm{~kW}, 0.9131$ p.u and 0.8250 , respectively. In which, the $V_{\min }$ is 0.0369 lower the permit range of the voltage amplitude. The network reconfiguration result by using the proposed MSA is presented in Table I. After reconfiguration by MSA, the switches $\{7-14-$ 9-32-28 $\}$ are opened instead of $\{33-34-35-36-37\}$. At that time, the total loss of the system is only $139.9823 \mathrm{~kW}$ that is reduced by $30.94 \%$ compared to the original network configuration. Furthermore, the $V_{\min }$ and $\left(I / I_{\text {rate }}\right)_{\max }$ have been enhanced. In more details, the $V_{\min }$ is 0.0281 higher than that of the initial configuration and the $\left(I / I_{\text {rate }}\right)_{\max }$ is lightly reduced from 0.8250 to 0.8126 after reconfiguration. The voltage and current of the 33-nodes system before and after reconfiguration by MSA are shown in Fig. 2. The figures demonstrate that most of node voltage amplitudes have been improved compared to the initial network configuration and the current of lines has been reduced after reconfiguration.

In comparisons with PSO and other previous methods, the result obtained by MSA is equal to that of PSO and the methods consisting of ACO [8], FWA [10]. While the methods comprising of IS-BPSO [13], ICSA [9], BBO [11], MPSO [12], MFPA [14], MOIWO [15], IAICA [20] and GA [3], the loss reduction obtained by MSA is $0.21 \%$ lower than that of the aforementioned methods but the minimum voltage amplitude is 0.0034 higher and violation of minimum voltage constraint at a smaller level than above methods. Compared to and HSA [18], the loss reduction obtained by MSA is $1.08 \%$ and $1.34 \%$ higher. In addition, the minimum voltage amplitude is also more improvement than the two above methods.

For the 69-nodes network, the Ploss, $V_{\min }$ and (I/ $\left.I_{\text {rate }}\right)_{\max }$ of the initial network configuration with open switches \{69-70-71-72-73\} are $224.8871 \mathrm{~kW}, 0.9092$ and 0.8767 , respectively. In which, the $V_{\min }$ is 0.0408 lower the permit range of the voltage amplitude. The network reconfiguration result by using the proposed MSA is presented in Table II. After reconfiguration by MSA, the switches \{6970-14-57-61\} are opened. This network configuration only causes $98.5875 \mathrm{~kW}$ that is reduced by $56.16 \%$ compared to the original network configuration. In addition, the $V_{\min }$ and $\left(I / I_{\text {rate }}\right)_{\max }$ have been improved. In which, the $V_{\min }$ is 0.0403 higher than that of the initial configuration and the (I/

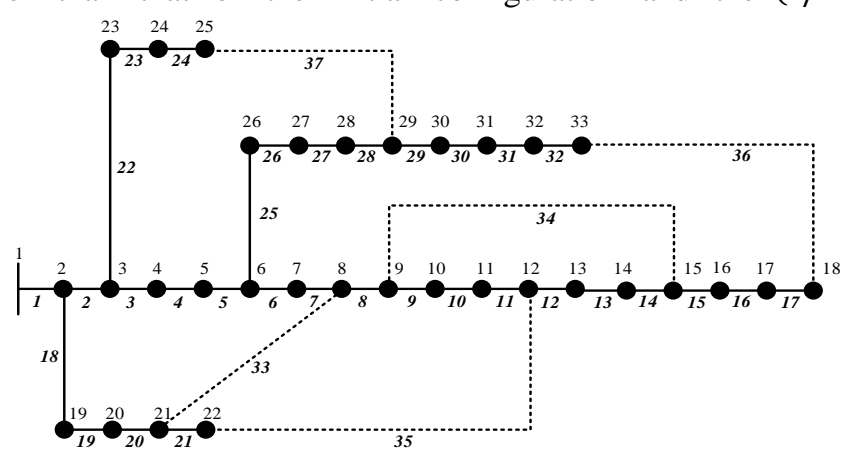

a) The 33-nodes test system
$\left.I_{\text {rate }}\right)_{\max }$ is lightly reduced from 0.8767 to 0.8572 after reconfiguration. The voltage and current of the 69-nodes system before and after reconfiguration by MSA are shown in Fig. 3. The figures send a message that the obtained benefits in enhancing voltage and current profiles after reconfiguration are noteworthy. Compared with PSO and other previous methods, the result obtained by MSA is identical to that of PSO and the methods consisting of ICSA [9] , ASFLA [16], BPSOGSA [17], FWA [10], BBO [11], GWO [21] and IAICA [20]. Compared to HSA [19], the loss reduction obtained by MSA is 2.93\% higher.

\section{B. The Performance of MSA}

The performance of MSA and PSO for the 33-nodes and 69-nodes system in 50 independent runs as presented in Table III. For the 33-nodes system, although both MSA and PSO have identified the optimal solution, MSA has found the best solution in 29 runs while PSO has only reached in the 5 runs in total of 50 independent trials. In addition, the maximum, average and standard deviations (STD) values of the fitness function in 50 runs of MSA are also much smaller than those of PSO. Specifically, these values of MSA are 161.0744, 150.6089 and 2.7915 which are much lower than 185.3416, 161.6831 and 9.3295 of PSO. The average number of converged iterations of MSA is higher than that of PSO, but obviously MSA usually converges to the optimal value while PSO is not. The calculation time of MSA is 1.0894 seconds faster than PSO. The maximum, minimum and average convergence curves of MSA and PSO for the 33-nodes system are presented in Fig. 4(a). From the figure, the average curve of MSA converges to lower value than that of PSO and the convergence value of the MSA's average curve is nearly equal to the convergence value of the smallest curve. This proves the superior efficiency of MSA compared to PSO for the NR problem.

The results obtained on a 69-nodes system are similar to those of the 33-nodes system. In 50 runs, the number of runs found the best solution of MSA is 20 while this number of PSO is only 5 runs that is 15 runs lower than compared to MSA. Similarly, the maximum, average and STD values of the fitness function in 50 runs of MSA are also much smaller than those of PSO. The calculation time of MSA for the 69-nodes system is 3.2731 seconds faster than PSO. The convergence curves of MSA shown in Fig. 4(b) are also send a message that MSA is more efficient and reliable than PSO for the NR problem.

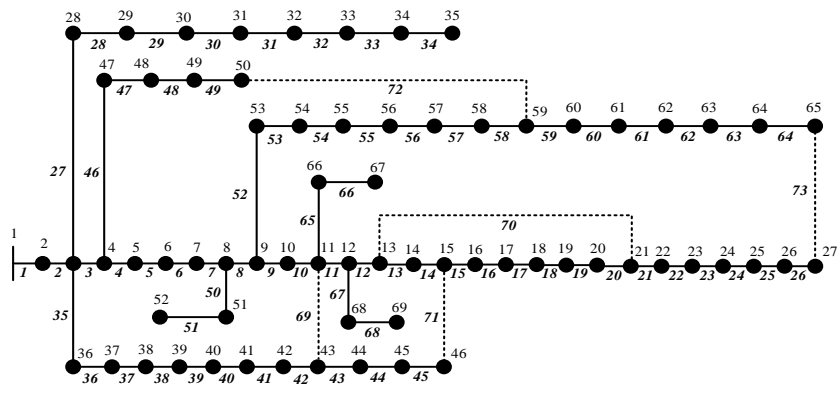

b) The 69-nodes test system

Fig. 1. The Test 33-Nodes and 69-Nodes Systems. 
TABLE I. THE RESUlts OF NETWORK RECONFIGURATION BY MSA FOR THE 33 NODES SYSTEM

\begin{tabular}{|l|l|l|l|l|l|}
\hline Method & Open switches & Ploss $(\mathbf{k W})$ & Reduction (\%) & $\boldsymbol{V}_{\min }(\mathbf{p . u})$ & 0.9131 \\
\hline Before rec. & $33-34-35-36-37$ & 202.6863 & - & 0.9412 & 0.8250 \\
\hline Proposed MSA & $7-14-9-32-28$ & 139.9823 & 30.94 & 0.9412 \\
\hline PSO & $7-14-9-32-28$ & 139.9823 & 30.94 & 0.9412 & 0.8126 \\
\hline ACO [8] & $7-14-9-32-28$ & 139.9823 & 30.94 & 0.9412 & - \\
\hline FWA [10] & $7-14-9-32-28$ & 139.9823 & 30.94 & 0.9378 & - \\
\hline IS-BPSO[13] & $7-14-9-32-37$ & 139.5500 & 31.15 & 0.9378 & - \\
\hline ICSA [9] & $7-14-9-32-37$ & 139.5500 & 31.15 & 0.9378 \\
\hline BBO [11] & $7-14-9-32-37$ & 139.5500 & 31.15 & 0.9378 & 0.8123 \\
\hline MPSO[12] & $7-14-9-32-37$ & 139.5500 & 31.15 & - \\
\hline MFPA [14] & $7-14-9-32-37$ & 139.5500 & 31.15 & 0.9378 \\
\hline IWO [15] & $7-14-9-32-37$ & 139.5500 & 31.15 & 0.9378 \\
\hline IAICA [20] & $7-14-9-32-37$ & 139.5500 & 31.15 & 0.9378 \\
\hline GA [3] & $7-14-9-32-37$ & 139.5500 & 31.15 & 0.9378 \\
\hline HSA [18] & $7-10-14-36-37$ & 142.6816 & 29.60 & - \\
\hline
\end{tabular}

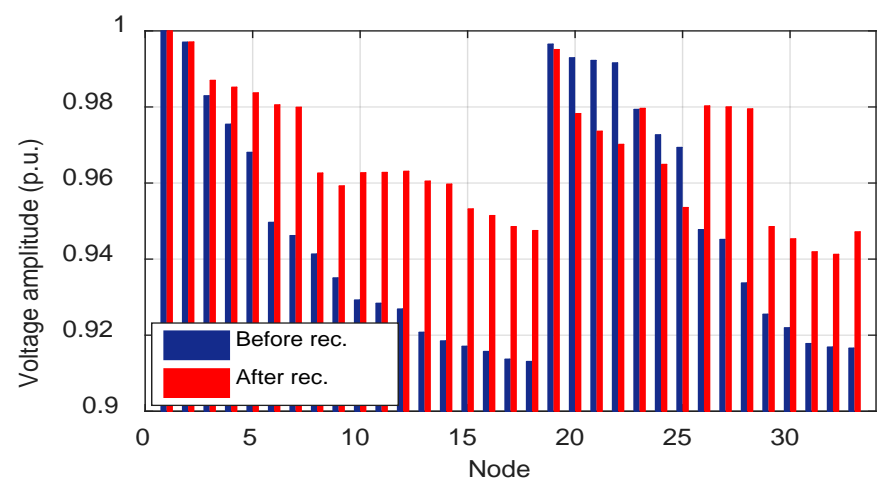

a) Voltage profile

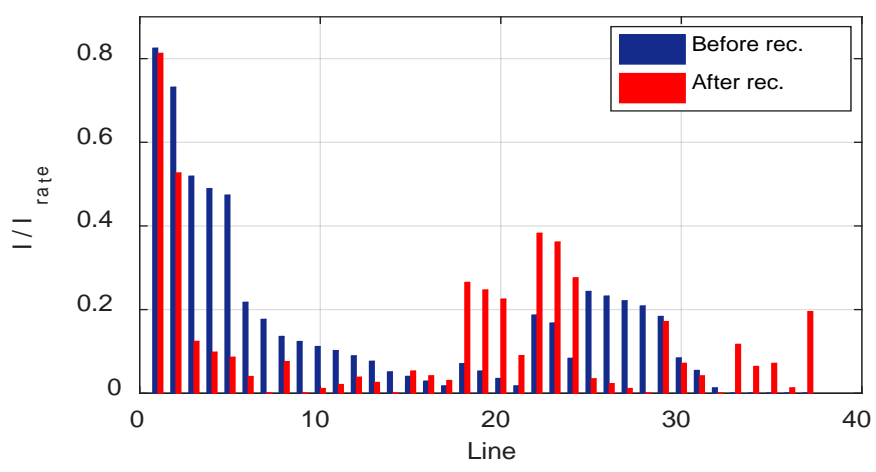

b) Current profile

Fig. 2. Voltages and Currents of the 33-Nodes System before and after Reconfiguration.

TABLE II. The Results of NETWORK RECONFIGURATION By MSA FOR THE 69 NODES SYSTEM

\begin{tabular}{|l|l|l|l|l|l|}
\hline Method & Open switches & Ploss (kW) & Reduction (\%) & $\boldsymbol{V}_{\min }(\mathbf{p . u})$ & $\left(\boldsymbol{I} / \boldsymbol{I}_{\text {rate }}\right)_{\max }$ \\
\hline Before rec. & $69-70-71-72-73$ & 224.8871 & - & 0.9092 & 0.8767 \\
\hline Proposed MSA & $69-70-14-57-61$ & 98.5875 & 56.16 & 0.9495 & 0.8572 \\
\hline PSO & $69-70-14-57-61$ & 98.5875 & 56.16 & 0.9495 & 0.8572 \\
\hline ICSA [9] & $14-57-61-69-70$ & 98.5875 & 56.16 & 0.9495 & - \\
\hline ASFLA [16] & $69-70-14-56-61$ & 98.5875 & 56.16 & 0.9495 & - \\
\hline BPSOGSA [17] & $69-70-14-56-61$ & 56.16 & 0.9495 & 0.9495 \\
\hline FWA [10] & $69-70-14-56-61$ & 56.5875 & 0.9427 & - \\
\hline BBO [11] & $14-70-69-58-61$ & 98.5875 & 56.16 & 0.9495 \\
\hline GWO [21] & $56-14-61-69-70$ & 98.5875 & 56.16 & 0.9495 \\
\hline IAICA [20] & $69-70-14-56-61$ & 98.5875 & 56.16 & - \\
\hline HSA [19] & $13-18-56-61-69$ & 98.5875 & 53.23 & - \\
\hline
\end{tabular}




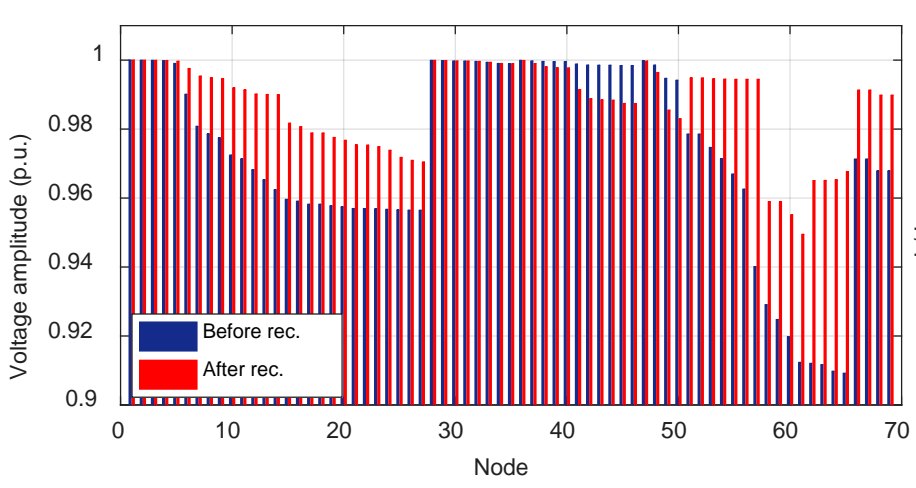

a) Voltage profile

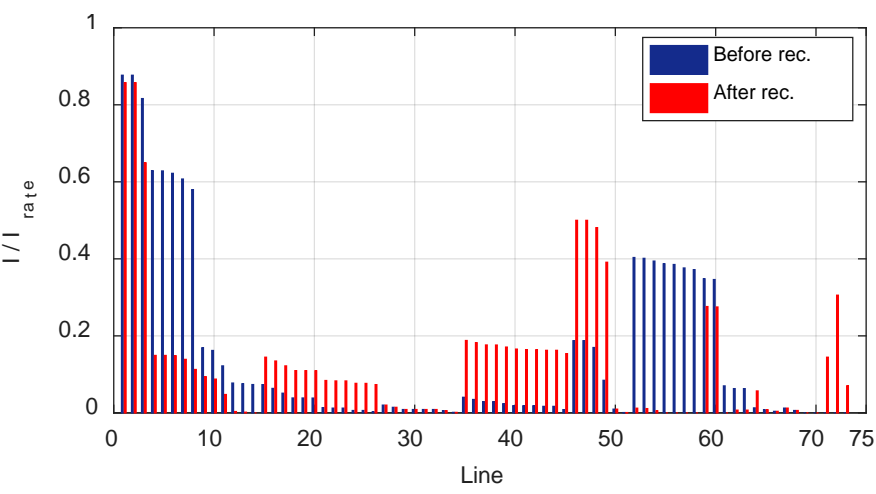

b) Current profile

Fig. 3. Voltages and Currents of the 69-Nodes System before and after Reconfiguration.

TABLE III. The Performance of the Proposed MSA For Two Test Systems

\begin{tabular}{|l|l|}
\hline Item & $\begin{array}{l}\text { MSA for the 33-node } \\
\text { system }\end{array}$ \\
\hline Optimal solution & $7-14-9-32-28$ \\
\hline Number of trials found the optimal solution & $29 / 50$ \\
\hline Maximum value of fitness function & 161.0744 \\
\hline Minimum value of fitness function & 148.7392 \\
\hline Average value of fitness function & 150.6089 \\
\hline STD value of fitness function & 2.7915 \\
\hline Maximum of convergence iterations & 149 \\
\hline Minimum of convergence iterations & 4 \\
\hline Average convergence iterations & 63.06 \\
\hline STD of convergence iterations & 48.3167 \\
\hline Execution times (second) & 8.3247 \\
\hline
\end{tabular}

a) For the 33 nodes system

\begin{tabular}{|l|l|l|}
\hline $\begin{array}{l}\text { PSO for the 33-nodes } \\
\text { system }\end{array}$ & $\begin{array}{l}\text { MSA for the 69-nodes } \\
\text { system }\end{array}$ & $\begin{array}{l}\text { PSO for the 69- } \\
\text { nodes system }\end{array}$ \\
\hline $7-14-9-32-28$ & $69-70-14-57-61$ & $69-70-14-57-61$ \\
\hline $5 / 50$ & $20 / 50$ & $12 / 50$ \\
\hline 185.3416 & 116.3852 & 140.7413 \\
\hline 148.7392 & 99.1169 & 99.1169 \\
\hline 161.6831 & 105.0324 & 114.1333 \\
\hline 9.3295 & 6.2900 & 15.1119 \\
\hline 67 & 150 & 76 \\
\hline 2 & 8 & 7 \\
\hline 22.84 & 79.06 & 35.18 \\
\hline 17.4313 & 50.1603 & 17.6412 \\
\hline 9.4141 & 25.2191 & 28.4922 \\
\hline
\end{tabular}

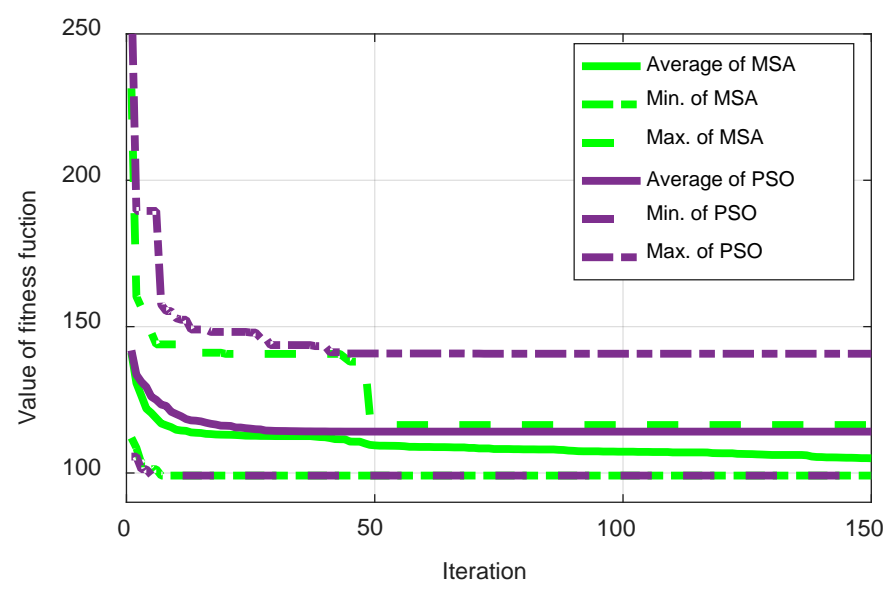

b) For the 69 nodes system

Fig. 4. Comparisons of Convergence Curves of MSA and PSO for Two Test Systems. 


\section{CONCLUSION}

The paper presents the network reconfiguration approach for the NR problem based on MSA. The goal of the NR problem is to find the best network configuration for minimizing power loss. The effectiveness of MSA is validated on two DS consisting of the 33-nodes and 69-nodes. The simulation results are compared to PSO and other available approaches in the literature. In comparison with PSO, MSA can determine the best network configuration with higher successful rate and better quality of obtained solution. In addition, MSA also outperforms to some previous approaches in the literature. Thus, MSA can be an effective and reliable tool for the NR problems. For future work, MSA can be used for the planning of practical distribution system operation.

\section{ACKNOWLEDGMENT}

This research was supported by Industrial University of Ho Chi Minh City under Grant No.37/HĐ-ĐHCN, signed on March, 2020.

\section{REFERENCES}

[1] Merlin and H. Back, "Search for a minimal loss operating spanning tree configuration in an urban power distribution system," Proceeding in 5th power system computation conf (PSCC), Cambridge, UK, vol. 1, pp. 118, 1975.

[2] K. Nara, A. Shiose, M. Kitagawa, and T. Ishihara, "Implementation of genetic algorithm for distribution systems loss minimum reconfiguration,” IEEE Transactions on Power Systems, vol. 7, no. 3, pp. 1044-1051, 1992, doi: 10.1109/59.207317.

[3] J. Z. Zhu, "Optimal reconfiguration of electrical distribution network using the refined genetic algorithm,” Electric Power Systems Research, vol. 62, no. 1, pp. 37-42, 2002, doi: 10.1016/S0378-7796(02)00041-X.

[4] A. Y. Abdelaziz, F. M. Mohammed, S. F. Mekhamer, and M. A. L. Badr, "Distribution Systems Reconfiguration using a modified particle swarm optimization algorithm,” Electric Power Systems Research, vol. 79, pp. 1521-1530, 2009, doi: 10.1016/j.epsr.2009.05.004.

[5] L. Li and C. Xuefeng, "Distribution Network Reconfiguration Based on Niche Binary Particle Swarm Optimization Algorithm,” Energy Procedia, vol. 17, pp. 178-182, 2012, doi: 10.1016/j.egypro.2012.02.080.

[6] A. Y. Abdelaziz, S. F. Mekhamer, F. M. Mohammed, and M. a L. Badr, "A Modified Particle Swarm Technique for Distribution Systems Reconfiguration," The online journal on electronics and electrical engineering(OJEEE), vol. 1, no. 1, pp. 121-129, 2009.

[7] R. Rao, S. V. L. Narasimham, and M. Ramalingaraju, "Optimization of distribution network configuration for loss reduction using artificial bee colony algorithm," World Academy of Science, Engineering and Technology, vol. 45, no. 2, 2008, [Online]. Available: http://citeseerx.ist. psu.edu/viewdoc/download?doi=10.1.1.192.9170\&rep=rep1\&type=pdf.

[8] Y. K. Wu, C. Y. Lee, L. C. Liu, and S. H. Tsai, "Study of reconfiguration for the distribution system with distributed generators," IEEE Transactions on Power Delivery, vol. 25, no. 3, pp. 1678-1685, 2010, doi: 10.1109/TPWRD.2010.2046339.

[9] T. T. Nguyen and T. T. Nguyen, "An improved cuckoo search algorithm for the problem of electric distribution network reconfiguration,” Applied Soft Computing, vol. 84, p. 105720, 2019, doi: 10.1016/j.asoc.2019. 105720.

[10] A. Mohamed Imran, M. Kowsalya, and D. P. Kothari, "A novel integration technique for optimal network reconfiguration and distributed generation placement in power distribution networks," International Journal of Electrical Power and Energy Systems, vol. 63, pp. 461-472, 2014, doi: 10.1016/j.jijepes.2014.06.011.

[11] M. Mosbah, R. Zine, S. Arif, and R. d. Mohammedi, "Optimum Distribution Network Reconfiguration in Presence DG Unit Using BBO Algorithm,” J. Electrical Systems, vol. 14, no. 4, pp. 180-189, 2018.
[12] F. M. F. Flaih, X. Lin, M. K. Abd, S. M. Dawoud, Z. Li, and O. S. Adio, "A new method for distribution network reconfiguration analysis under different load demands,” Energies, vol. 10, no. 4, pp. 1-19, 2017, doi: 10.3390/en10040455.

[13] R. Pegado, Z. Ñaupari, Y. Molina, and C. Castillo, "Radial distribution network reconfiguration for power losses reduction based on improved selective BPSO," Electric Power Systems Research, vol. 169, no. December 2018, pp. 206-213, 2019, doi: 10.1016/j.epsr.2018.12.030.

[14] S. Ganesh and R. Kanimozhi, "Meta-heuristic technique for network reconfiguration in distribution system with photovoltaic and DSTATCOM,” IET Generation, Transmission \& Distribution, vol. 12, no. 20, pp. 4524-4535, 2018, doi: 10.1049/iet-gtd.2018.5629.

[15] D. Sudha Rani, N. Subrahmanyam, and M. Sydulu, "Multi-Objective Invasive Weed Optimization - An application to optimal network reconfiguration in radial distribution systems," International Journal of Electrical Power \& Energy Systems, vol. 73, pp. 932-942, 2015, doi: 10.1016/j.ijepes.2015.06.020.

[16] A. Onlam, D. Yodphet, R. Chatthaworn, C. Surawanitkun, A. Siritaratiwat, and P. Khunkitti, "Power Loss Minimization and Voltage Stability Improvement in Electrical Distribution System via Network Reconfiguration and Distributed Generation Placement Using Novel Adaptive Shuffled Frogs Leaping Algorithm,” Energies, vol. 12, no. 3, p. 553, 2019, doi: 10.3390/en12030553.

[17] A. Fathy, M. El-Arini, and O. El-Baksawy, "An efficient methodology for optimal reconfiguration of electric distribution network considering reliability indices via binary particle swarm gravity search algorithm," Neural Computing and Applications, 2017, doi: 10.1007/s00521-0172877-z.

[18] R. Srinivasa Rao, S. V. L. Narasimham, M. Ramalinga Raju, and A. Srinivasa Rao, "Optimal Network Reconfiguration of Large-Scale Distribution System Using Harmony Search Algorithm,” IEEE Transactions on Power Systems, vol. 26, no. 3, pp. 1080-1088, Aug. 2011, doi: 10.1109/TPWRS.2010.2076839.

[19] R. S. Rao, K. Ravindra, K. Satish, and S. V. L. Narasimham, "Power Loss Minimization in Distribution System Using Network Recon figuration in the Presence of Distributed Generation,” IEEE Transaction on Power System, vol. 28, no. 1, pp. 317-325, 2013, doi: 10.1109/TPWRS.2012.2197227.

[20] S. H. Mirhoseini, S. M. Hosseini, M. Ghanbari, and M. Ahmadi, "A new improved adaptive imperialist competitive algorithm to solve the reconfiguration problem of distribution systems for loss reduction and voltage profile improvement," International Journal of Electrical Power and Energy Systems, vol. 55, pp. 128-143, 2014, doi: 10.1016/j.ijepes.2013.08.028.

[21] A. V. S. Reddy, M. D. Reddy, and M. S. K. Reddy, "Network reconfiguration of distribution system for loss reduction using GWO algorithm,” International Journal of Electrical and Computer Engineering, vol. 7, no. 6, pp. 3226-3234, 2017, doi: 10.11591/ijece.v7i6.pp32263234.

[22] A. A. A. Mohamed, Y. S. Mohamed, A. A. M. El-Gaafary, and A. M. Hemeida, "Optimal power flow using moth swarm algorithm," Electric Power Systems Research, vol. 142, pp. 190-206, 2017, doi: 10.1016 /j.epsr.2016.09.025.

[23] A. Mohamed, "Moth Swarm Algorithm (MSA).” MATLAB Central File Exchange, 2020, [Online]. Available: https://www.mathworks.com/ matlabcentral/fileexchange/57822-moth-swarm-algorithm-msa.

[24] A. Y. Abdelaziz, F. M. Mohamed, S. F. Mekhamer, and M. A. L. Badr, "Distribution system reconfiguration using a modified Tabu Search algorithm,” Electric Power Systems Research, vol. 80, no. 8, pp. 943953, 2010, doi: 10.1016/j.epsr.2010.01.001.

[25] M. E. Baran and F. F. Wu, "Network reconfiguration in distribution systems for loss reduction and load balancing," IEEE Transactions on Power Delivery, vol. 4, no. 2. pp. 1401-1407, 1989, doi: 10.1109/61.25627.

[26] H.-D. Chiang and R. Jean-Jumeau, "Optimal network reconfigurations in distribution systems: Part 2: Solution algorithms and numerical results," IEEE Transactions on Power Delivery, vol. 5, no. 3, pp. 1568-1574, 1990, doi: 10.1109/61.58002. 